\title{
Article \\ A Gated Oscillator Clock and Data Recovery Circuit for Nanowatt Wake-Up and Data Receivers
}

\author{
Matteo D'Addato ${ }^{1,2, *}$, Alessia M. Elgani ${ }^{1,2} \oplus$, Luca Perilli ${ }^{2} \oplus$, Eleonora Franchi Scarselli ${ }^{1,2}$, \\ Antonio Gnudi ${ }^{1,2}{ }^{\mathbb{D}}$, Roberto Canegallo ${ }^{3}$ and Giulio Ricotti ${ }^{3}$ \\ 1 Electrical, Electronic and Information Engineering Department "Guglielmo Marconi", University of Bologna, \\ Viale Risorgimento 2, 40123 Bologna, Italy; alessiamaria.elgani@unibo.it (A.M.E.); \\ eleonora.franchi@unibo.it (E.F.S.); antonio.gnudi@unibo.it (A.G.) \\ 2 Advanced Research Center on Electronic Systems "Ercole De Castro", University of Bologna, \\ 40123 Bologna, Italy; luca.perilli@unibo.it \\ 3 STMicroelectronics, 20864 Agrate Brianza e Cornaredo, Italy; roberto.canegallo@st.com (R.C.); \\ giulio.ricotti@st.com (G.R.) \\ * Correspondence: matteo.daddato2@unibo.it
}

check for updates

Citation: D'Addato, M.; Elgani, A.M.; Perilli, L.; Franchi Scarselli, E.; Gnudi, A.; Canegallo, R.; Ricotti, G. A Gated Oscillator Clock and Data Recovery Circuit for Nanowatt Wake-Up and Data Receivers. Electronics 2021, 10, 780 . https://doi.org/10.3390/ electronics10070780

Academic Editor: Inhee Lee

Received: 28 February 2021

Accepted: 22 March 2021

Published: 25 March 2021

Publisher's Note: MDPI stays neutral with regard to jurisdictional claims in published maps and institutional affiliations.

Copyright: (c) 2021 by the authors. Licensee MDPI, Basel, Switzerland. This article is an open access article distributed under the terms and conditions of the Creative Commons Attribution (CC BY) license (https:/ / creativecommons.org/licenses/by/ $4.0 /)$.

\begin{abstract}
This article presents a data-startable baseband logic featuring a gated oscillator clock and data recovery (GO-CDR) circuit for nanowatt wake-up and data receivers (WuRxs). At each data transition, the phase misalignment between the data coming from the analog front-end (AFE) and the clock is cleared by the GO-CDR circuit, thus allowing the reception of long data streams. Any free-running frequency mismatch between the GO and the bitrate does not limit the number of receivable bits, but only the maximum number of equal consecutive bits $\left(N_{m}\right)$. To overcome this limitation, the proposed system includes a frequency calibration circuit, which reduces the frequency mismatch to $\pm 0.5 \%$, thus enabling the WuRx to be used with different encoding techniques up to $N_{m}=100$. A full WuRx prototype, including an always-on clockless AFE operating in subthreshold, was fabricated with STMicroelectronics $90 \mathrm{~nm} \mathrm{BCD}$ technology. The WuRx is supplied with $0.6 \mathrm{~V}$, and the power consumption, excluding the calibration circuit, is $12.8 \mathrm{nW}$ during the rest state and $17 \mathrm{nW}$ at a $1 \mathrm{kbps}$ data rate. With a $1 \mathrm{kbps}$ On-Off Keying (OOK) modulated input and $-35 \mathrm{dBm}$ of input RF power after the input matching network (IMN), a $10^{-3}$ missed detection rate with a 0 bit error tolerance is measured, transmitting 63 bit packets with the $N_{m}$ ranging from 1 to 63 . The total sensitivity, including the estimated IMN gain at $100 \mathrm{MHz}$ and $433 \mathrm{MHz}$, is $-59.8 \mathrm{dBm}$ and $-52.3 \mathrm{dBm}$, respectively. In comparison with an ideal CDR, the degradation of the sensitivity due to the GO-CDR is $1.25 \mathrm{dBm}$. False alarm rate measurements lasting $24 \mathrm{~h}$ revealed zero overall false wake-ups.
\end{abstract}

Keywords: clock and data recovery (CDR); Internet of things (IoT); nanowatt data receiver; ultralow-power; wake-up receiver (WuRx)

\section{Introduction}

Energy efficiency is a fundamental metric for all battery-powered devices, such as wireless sensor and actuator network (WSAN) nodes, whose most power-hungry subsystem is usually the RF transceiver. A wake-up receiver (WuRx) is an always-on ultra-low-power receiver which constantly monitors the channel and wakes the node up at the reception of a communication request in order to overcome the trade-off between power consumption and node latency $[1,2]$. WuRxs can be classified depending on their application range. Short-range WuRxs are fully passive and achieve a communication distance limited to few centimeters or meters. Medium-range WuRxs are used in applications requiring a range of, at most, $100 \mathrm{~m}$, their power consumption typically being in the nanowatt range. Long-range $\mathrm{WuRxs}$ consume microwatts and can receive packets from kilometers away.

A typical WuRx architecture is composed of two subsystems: an analog front-end (AFE) and a baseband logic. The AFE turns the RF input's OOK-modulated signal into a 
stream of bits, whereas the baseband logic generates the wake-up signal upon reception of the correct codeword [3-9]. State-of-the-art ultra-low-power WuRxs use oversampling techniques to overcome the phase alignment problem between the received data and internal clock and, with the aim of limiting the power consumption to a few nanowatts, typically use ring or relaxation oscillators. The frequency accuracy of such oscillators is poor (only a few percent), thus limiting the maximum receivable packet length from 8 bits to 63 bits. In Reference [8], to increase the WuRx sensitivity and minimize the number of false wake-ups due to the noise and uncertainty of the clock frequency, a $2 x$ oversampling scheme and a relaxation oscillator were employed, and an optimal 16 bit code was designed. This was unlike the applications in [8,9], which proposed a WuRx able to receive a set of different and longer packets ( $63 \mathrm{bits})$. This feature would enable it to also transmit encrypted data, which is a key issue for enhancing the security of WSANs [9-12]. The solution proposed in [9] included a ring oscillator and a $4 x$ oversampling architecture designed to tolerate 13 errors in the received packet, which implied a higher number of false wake-ups compared with [8]. Similar oversampling techniques were employed in recently proposed $\mathrm{WuRxs}[12,13]$. To allow the WuRx to receive long packets with no constraints in terms of false wake-ups, as proposed in [3], it is possible to employ oversampling circuits in which data sampling is performed using crystal oscillators. They ensure excellent frequency stability and the capability of receiving long data. This is carried out at the cost of a power consumption far above tens of nanowatts, which is not affordable for ultra-low-power WuRxs. As an alternative, it is possible to employ clock and data recovery (CDR) circuits based on Phase Locked Loops (PLLs) [14]. They ensure phase and frequency alignment between the received data and the clock, with a power consumption far lower than that required by crystal oscillators. However, PLLs need long preamble times (tens of bit times) to settle the clock frequency according to the received data rate, which is not acceptable in the case where the WuRx must also be employed for burst communications. A WuRx with an injection-locked oscillator (ILO) CDR, which guarantees lower preamble times, was proposed in [15]. Nevertheless, [15] needed Manchester encoding for the received data, which implies a halving in the data rate to prevent the ILO from going back to its free running mode due to the absence of data transitions. Recently, [16] proposed a wake-up and data receiver in which the sampling time selection was achieved through a digitally programmable interface, while frequency control was carried out using a frequency-locked loop (FLL). Similar to [14], it required a non-negligible time to set the clock frequency.

To overcome the issues related to the power consumption, maximum packet length, false wake-up tolerance, preamble time and data encoding, in [11], a nanowatt WuRx suitable for receiving infinite bits in addition to a codeword targeting medium-range applications at a $1 \mathrm{kbps}$ data rate was proposed. The phase alignment between the received data and the clock was carried out in [11] using a CDR based on a gated oscillator (GO), which guaranteed a short preamble time and ultra-low power consumption. A similar synchronization scheme was employed in [17], where the limitation on the maximum number of equal consecutive bits was not taken into account.

In this paper, we present an implementation in STMicroelectronics $90 \mathrm{~nm}$ BCD technology and the experimental results of a nanowatt $\mathrm{WuRx}$, enabling the transmission of long codes based on the GO-CDR architecture we proposed in [11] and including an AFE with MOSFETs operating in the subthreshold region and a calibration circuit for the GO-CDR, which allows the WuRx to process data containing even long streams of equal consecutive bits. The target is a $1 \mathrm{kbps}$ data rate for an OOK-modulated input RF signal.

The remainder of this paper is organized as follows. Section 2 describes the proposed WuRx architecture with special emphasis on the baseband logic. Sections 3 and 4 present the circuit design and the implementation choices, respectively. Section 5 shows the measurement results, and finally, Section 6 concludes the paper. 


\section{Wake-Up and Data Receiver Architecture}

The proposed WuRx is shown in Figure 1. The always-on AFE was clockless (i.e., it did not need an oscillator), while the baseband logic required a clock to sample the incoming data. This allowed the WuRx to operate in two phases. During Phase 1, the baseband logic was off, whereas the AFE was active. Phase 2 started upon recognition of the first 0-to-1 transition of the message, occurring at the first transition of the AFE output signal. The baseband logic was turned on, and the incoming bitstream was compared with the stored codeword. This approach allowed us to reduce the power consumption of the node if the specific application was characterized by long idle periods, since the baseband logic was off most of the time [16].

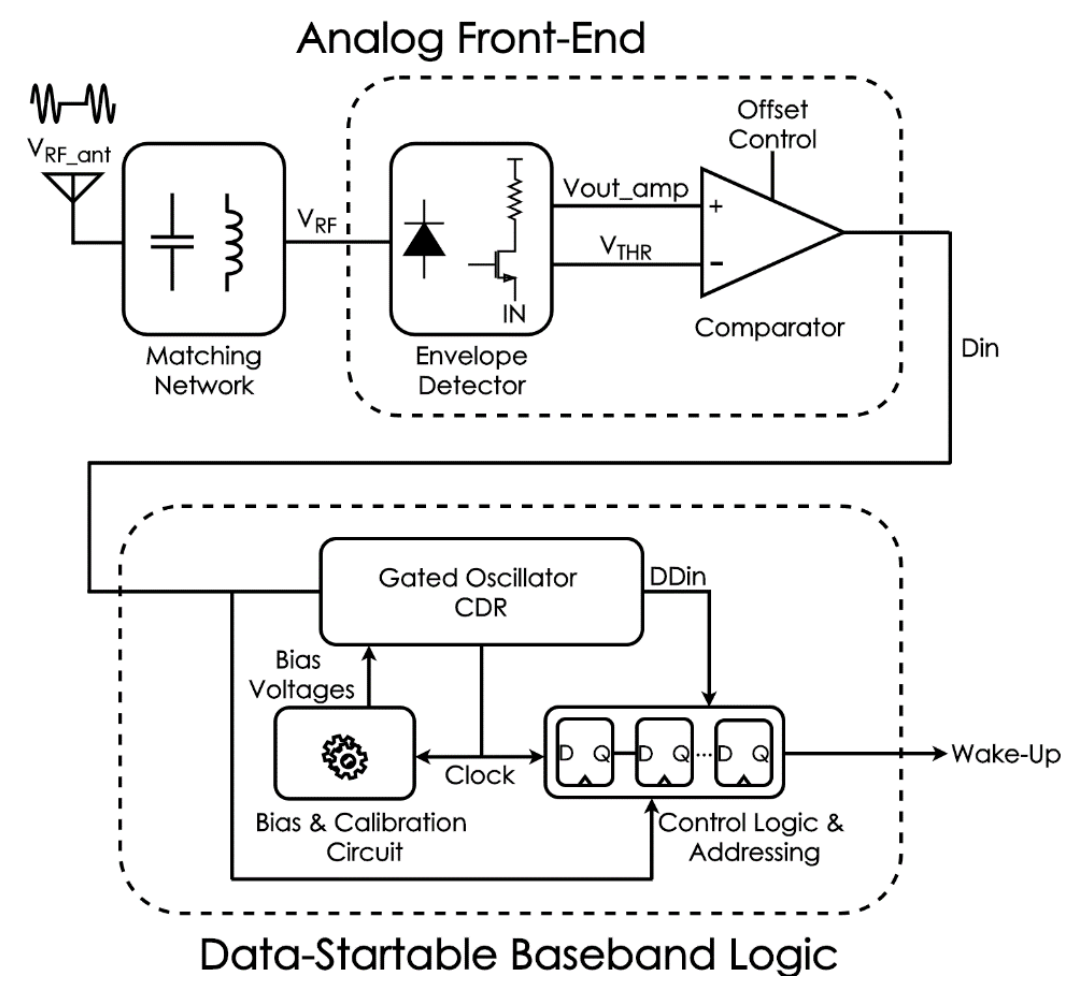

Figure 1. Block diagram of the proposed wake-up and data receiver (WuRx).

The AFE was composed of an external lumped component input matching network (IMN) followed by an envelope detector (ED) and a comparator, both of which were integrated on the chip.

As indicated in Figure 1, the proposed data-startable baseband logic included [11] (1) a GO-CDR, (2) a control logic with addressing capabilities (CL) to generate the wakeup signal and control signals for GO-CDR and (3) a bias and calibration (BC) circuit for the GO-CDR.

As illustrated in Figure 2a, the purpose of the CDR circuit was to provide a clock to the $\mathrm{CL}$ to correctly sample on the positive edges a delayed version of the input data (DDin). As shown in Figure 2b, ideally, the sampling edges would be placed at the center of each bit time $\left(T_{b}\right)$. The circuit was composed of three sections: a delay block (DB), an edge detector implemented through an Exclusive NOR (EXNOR) gate and the GO. The EXNOR gate was fed with the data signal, Din, and its delayed version, DDin, resulting in a pulse of the gate signal at each Din transition. When Gate $=1$, the GO was in free running mode with a frequency $f_{c k}=1 / T_{c k}$, while with Gate $=0$, it was blocked in a predefined state. When Gate switched from 0 to 1 , the GO generated the positive edge of Clock, ideally after $T_{c k} / 2$, thus allowing it to clear any phase error accumulated up to that time, even if the free-running clock frequency was not precisely matched to the data rate $\left(T_{c k} \neq T_{b}\right)$. Therefore, the only constraint of this architecture is on the maximum number of equal consecutive bits $\left(N_{m}\right)$ 
that can be correctly sampled. $N_{m}$ can be calculated, imposing that no bit is sampled twice (which can occur if $T_{c k}<T_{b}$ ) or not sampled at all (which can occur if $T_{c k}>T_{b}$ ). Defining $\alpha=\left|T_{c k}-T_{b}\right| / T_{b}$, a simplified analysis carried out assuming a start-up time of zero for the oscillator and neglecting the Clock jitter, leads to the following constraints:

$$
\begin{gathered}
\frac{T_{c k}}{2}+N_{m} T_{c k}>N_{m} T_{b} \text { if } T_{c k}=T_{b}(1-\alpha) \\
\frac{T_{c k}}{2}+N_{m} T_{c k}<\left(N_{m}+1\right) T_{b} \text { if } T_{c k}=T_{b}(1+\alpha)
\end{gathered}
$$

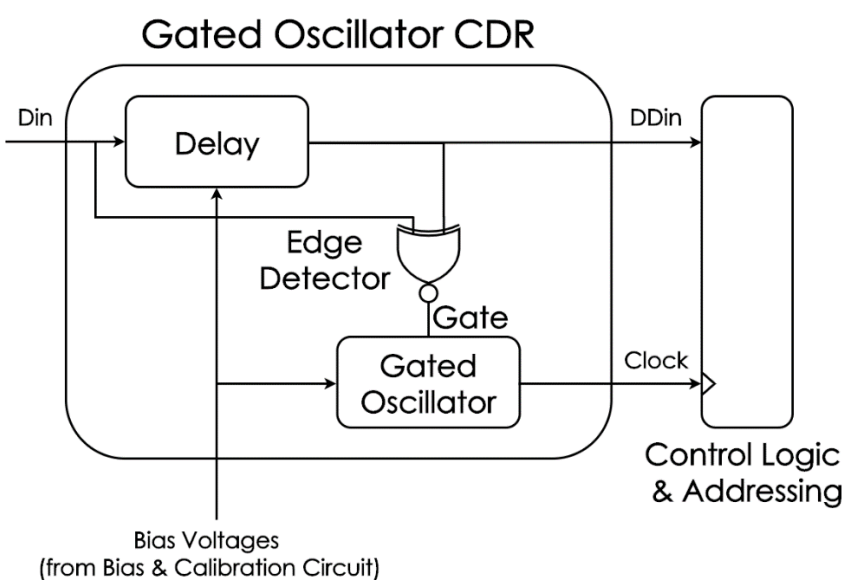

(a)

Ideal case: $T_{c k}=T_{b}$

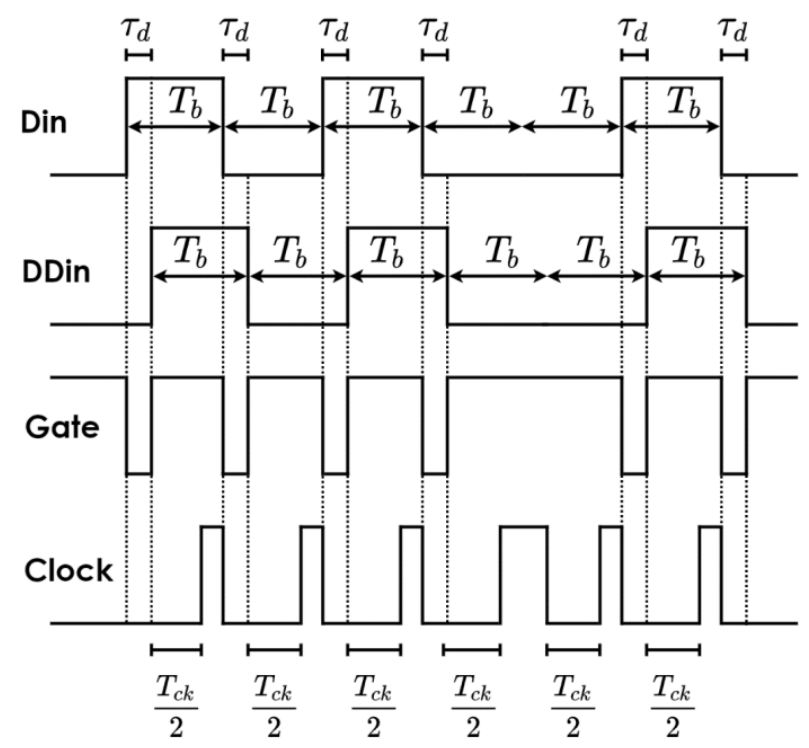

(b)

Figure 2. (a) Gated oscillator clock and data recovery (GO-CDR) circuit. (b) GO-CDR circuit behavior. $T_{c k}$ is the clock period, $T_{b}$ is the bit-time and $\tau_{d}$ is the delay between the input data (Din) and the delayed version of the input data (DDin).

This results in $N_{m}<(1-\alpha) /(2 \alpha)$ [11].

In case a Manchester code is employed, which contains a transition in each bit time (i.e., $N_{m}=2$ ) at the cost of halving the data rate compared with the standard binary encoding, the equation leads to $\alpha<0.2$. Such a frequency error upper limit is easily achievable in integrated ultra-low-power oscillators. 
To avoid the use of a Manchester code with its associated limitations on the data rate, the proposed architecture included a bias and calibration circuit for the GO-CDR, which reduced $\alpha$ to negligible values and then allowed the WuRx to process data containing long sequences of equal consecutive bits.

\section{Circuit Design}

\subsection{Analog Front-End}

The AFE of the implemented WuRx (Figure 1) was composed of an envelope detector, which simultaneously extracted the envelope of the incoming OOK signal and amplified it at the baseband, a comparator with a variable threshold to digitize the extracted envelope and a reference current generator to provide bias currents for both the ED and the comparator. The circuit schematic of the ED, shown in Figure 3a, was an elaboration of that in [18], where subthreshold operation allowed envelope extraction, leveraging second-order nonlinearities. The self-biasing scheme for the gain transistor M5 allowed for setting a robust DC operating point. The correct operation required the Resistance-Capacitance (RC) time constant chosen to be large enough to maintain the gate voltage of M5, $V_{\mathrm{REF}}$, almost equal to its quiescent value (corresponding to the zero RF input signal) also during the reception of an entire packet. If this condition was met, M5 effectively operated as a common gate amplifier. Correspondingly, the high and low values of $\mathrm{V}_{\text {OUT_AMP }}$ remained constant throughout the whole packet, as shown in the inset of Figure 3a, and the output voltage $\mathrm{V}_{\text {OUT_AMP }}$ was a low-pass filtered version of the RF input envelope. This guaranteed the correct operation of the comparator with a fixed threshold. More details can be found in [18]. Unlike the solution in [18], no cascode transistor was employed in the ED, thus allowing the use of a supply voltage lower than the nominal one, leading to a reduction in power consumption.

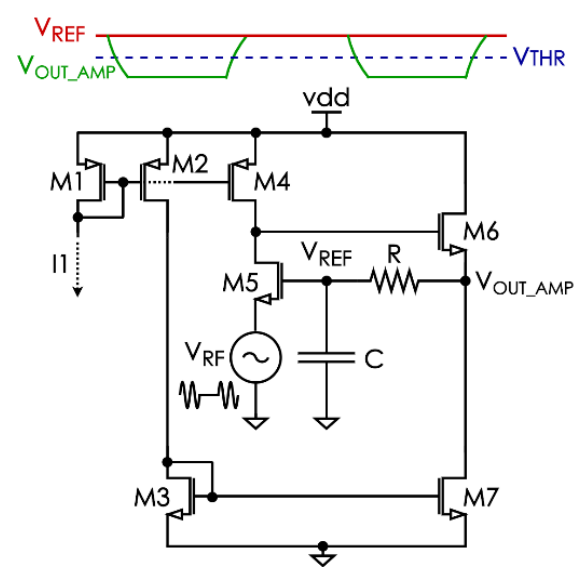

(a)

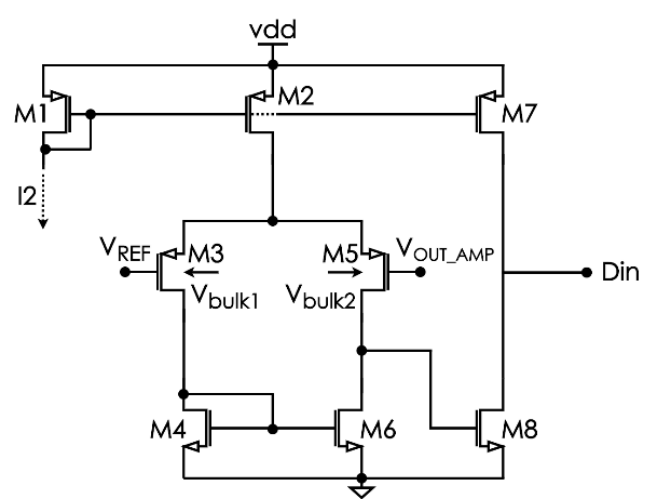

(b)

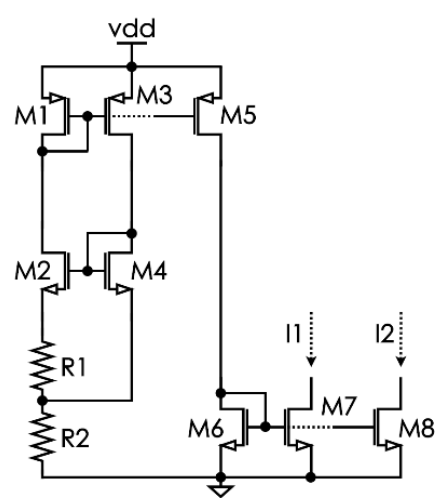

(c)

Figure 3. (a) Schematic of the envelope detector (ED). The inset shows a qualitative time-domain response to a 1-0-1 input sequence in green, as well as the gate voltage of $\mathrm{M} 5, \mathrm{~V}_{\mathrm{REF}}$, in red and the effective threshold of the comparator in blue. (b) Schematic of the comparator. (c) Schematic of the biasing block.

The comparator schematic is shown in Figure $3 \mathrm{~b}$. It received both the ED output voltage and the voltage at the gate of M5 which, as said above, remained almost constant at its quiescent value for the entire packet reception. The body effect of the differential pair transistors (M3 = M5) was exploited to set the effective threshold of the comparator $\mathrm{V}_{\mathrm{THR}}$ by adjusting the externally supplied bulk voltages $\mathrm{V}_{\text {BULK1 }}$ and $\mathrm{V}_{\text {BULK2 }}$. The inset of Figure 3 a shows the relationships between $V_{\text {OUT_AMP }}, V_{\text {REF }}$ and $V_{T H R}$.

Both the ED and the comparator were biased with Proportional-to-Absolute-Temperature (PTAT) currents [19] generated by the circuit in Figure 3c. The effectiveness of using a PTAT current for a more constant ED gain within the $-20-85^{\circ} \mathrm{C}$ temperature range has been proven through simulations [18]. 


\subsection{Gated Oscillator and Delay Block}

The GO is shown in Figure 4. It was a ring oscillator composed of three stages, each of which consisted of a current-starved inverter (CSI) (M1-M4, M7-M10 and M13-M16), a capacitor (C1, C2 and C3) and two additional transistors (M5-M6, M11-M12 and M17-M18) driven by the Gate signal to reset the output of each CSI (O1, O2 and O3) to a predefined state at each pulse in the Gate signal.

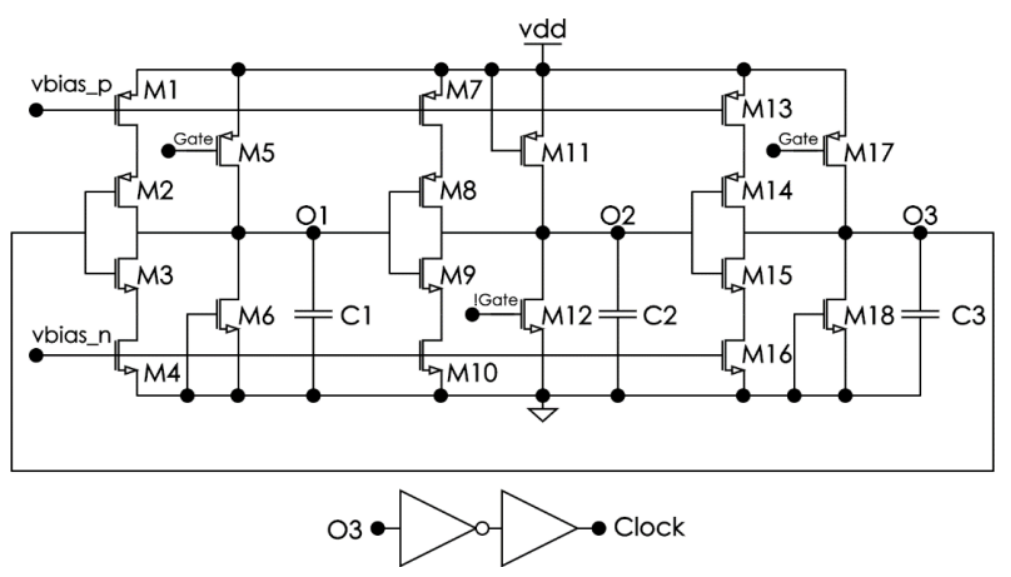

Figure 4. Schematic of the gated oscillator (GO).

The output of the GO was fed to an inverting stage to generate a squared clock signal. The oscillation frequency was $1 /\left(2 N \tau_{p}\right)$, where $N=3$ was the number of stages and $\tau_{p}$ was the propagation delay of each stage, yielding $\tau_{p}=T_{c k} / 6$. Bias voltages vbias_p and vbias_n controlled the charging and discharging currents for capacitors C1, C2 and C3 and thus the value of $\tau_{p}$. The delay block DB consisted of a stage equal to the ones used in the GO biased by the same control voltages vbias_p and vbias_n (with the two additional transistors biased as off) followed by an inverting stage to square its output signal (DDin). These choices ensured $\tau_{d}=\tau_{p}$ (where $\tau_{d}$ is the delay between Din and DDin), which implied that the necessary condition $\tau_{d}<T_{b} / 2$ was always satisfied [11]. This condition prevented the clock's high phase from having a null duration. Furthermore, $\tau_{d}$ must be a chosen value larger than the reset time $\left(\tau_{\text {res }}\right)$ of the oscillator. The design constraints on the value of $\tau_{d}$ are therefore

$$
\tau_{\text {res }}<\tau_{d}<T_{b} / 2
$$

\subsection{Control Logic with Addressing Capabilities}

The CL is shown in Figure 5. It was composed of four blocks: (1) a serial-input paralleloutput (SIPO) register, (2) a correlator with a programmable codeword and threshold (adapted from [8]), (3) a programmable timeout counter and (4) a sequential unit (SU). The configuration parameters (i.e., the codeword, correlator threshold and timeout values), were assigned to the CL by programming the SIPO register. Figure 6 summarizes the behavior of the CL. The SU detected a 0-to-1 transition of the Din and forced the WuRx into Phase 2. When the GO-CDR was activated, the generated clock was used by the CL to sample the incoming bitstream (DDin). In particular, the SU detected a start frame delimiter (SFD), which enabled the correlator to start the comparison between the DDin and the codeword (en_corr switches from 0 to 1 ). The CL generated the wake-up signal only when the correlation result was higher than the threshold of the correlator. The CL also included a timeout counter, triggered by the clock provided by GO-CDR, to push the system back to Phase 1 after a predefined time interval elapsed without detecting the correct codeword.

The assignment of the configuration parameters was crucial to optimizing the performance of the entire system. In particular, the correlator threshold together with the codeword length could be set as a function of the sensitivity of the WuRx in order to reduce 
the number of false wake-ups in noisy environments. Consistently, the timeout value could be set accordingly to reduce power consumption during Phase 2 .

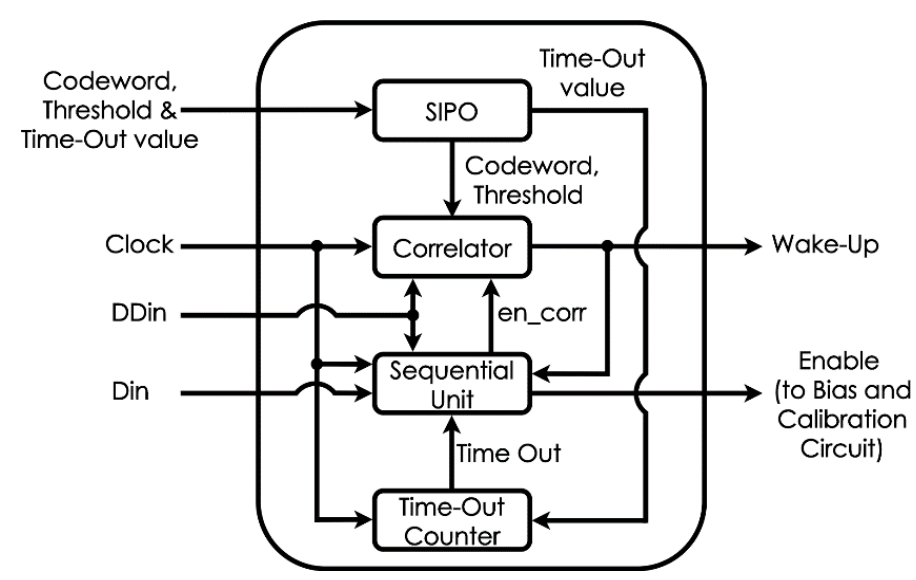

Figure 5. Schematic of the control logic with addressing capabilities (CL).

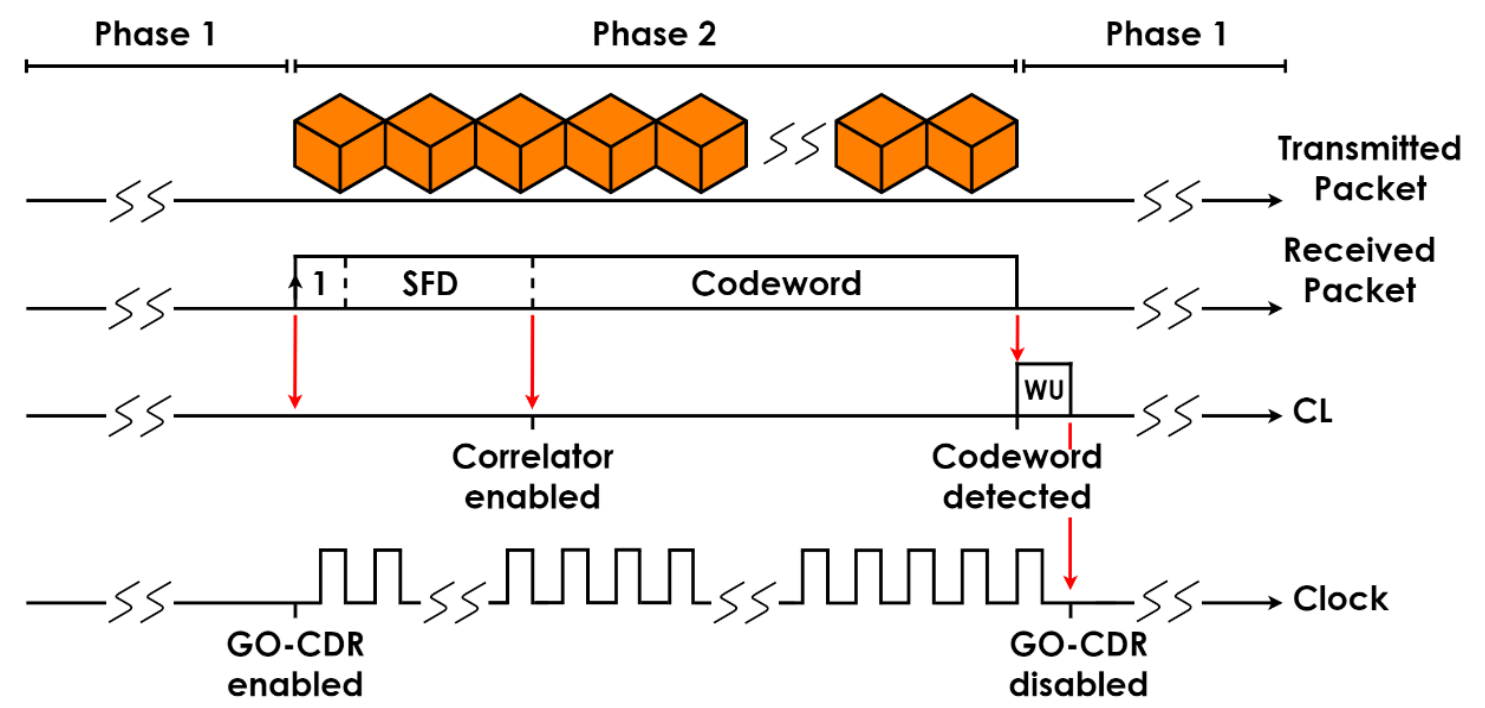

Figure 6. Behavior of the control logic with addressing capabilities (CL). WU is the wake-up signal.

\subsection{Bias and Calibration Circuit}

The block diagram of the bias and calibration (BC) circuit is shown in Figure 7. It was composed of a frequency detector (FD) adapted from [20], a successive approximation logic (SA Logic) and a digital controlled current source (DCCS). The BC circuit was in charge of generating the bias voltages (vbias_p and vbias_n) for the GO-CDR so that the oscillation frequency of the GO was equal to the target data rate, even with process, voltage and temperature (PVT) variations. The FD detected the frequency difference between the clock and the external reference (Clock_ref) equal to the data rate, while the SA Logic was used to set the bits (bi_UP-bi_DN) of the DCCS, exploiting the output signals (UP-DN) of the FD. In particular, the DCCS generated the bias voltages vbias_p and vbias_n for GO-CDR using binary weighted currents. For testing purposes, in the present implementation, these currents were generated from an external current source (Ibias). If the frequency of clock was too close or too far from the Clock_ref, the generation of UP-DN pulses could require many clock cycles or could not occur at all. To avoid the stall condition, the SA Logic included a counter, which forced the end of the calibration in case its timeout value was reached (i.e., End_Calib switches from 0 to 1). 


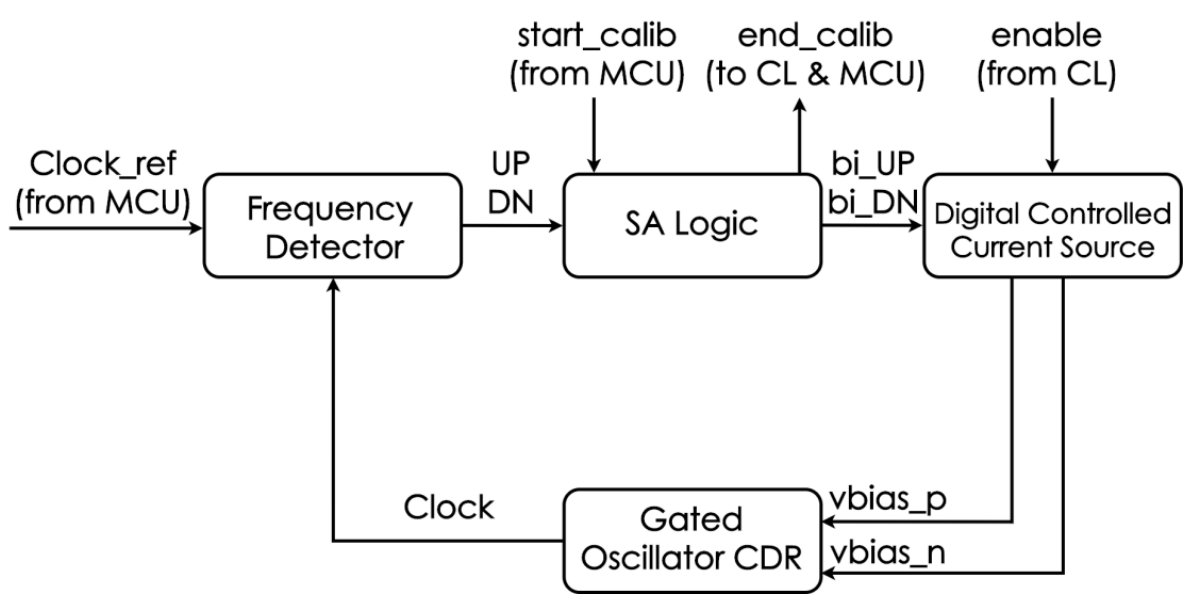

Figure 7. Block diagram of the bias and calibration (BC) circuit.

The calibration started switching from 0 to 1 the Start-Calib signal to enable the SA Logic and force the CL to trigger GO-CDR using the Enable signal. At the same time, Clock_ref was applied to the FD. The calibration ended when the least significant bit of the DCCS was set (i.e., End_Calib switches from 0 to 1) or, as mentioned above, when the counter reached the timeout value. In particular, the calibration cycle was managed by the node Microcontroller Unit (MCU), which had to generate the Clock_ref and start_calib signals for BC. The power consumption of Clock_ref was negligible, since the calibration procedure could be activated only in few cases: (1) when the node was started up, (2) at predefined time steps and (3) when the temperature of the node was higher or lower than the predefined thresholds.

\section{Implementation Choices}

The proposed WuRx was designed using STMicroelectronics $90 \mathrm{~nm}$ BCD technology, targeting a $1 \mathrm{kbps}$ bitrate. The AFE, the GO-CDR and the CL were designed to have a $0.6 \mathrm{~V}$ supply voltage (vdd), whereas in the current prototype, the BC circuit was designed for operation with a standard $1.2 \mathrm{~V}$ supply.

\subsection{Analog Front-End}

The ED (Figure 3a) was biased with $\mathrm{I}_{1}=1 \mathrm{nA}$. The first pole was due to an integrated $75 \mathrm{M} \Omega$ resistor in series with an output resistance of M6 (roughly $75 \mathrm{M} \Omega$ ) and an external $500 \mathrm{nF}$ capacitance. The comparator (Figure $3 \mathrm{~b}$ ) was biased with $\mathrm{I}_{2}=1 \mathrm{nA}$ as well. As mentioned in Section 3, the bulk voltages of the transistors belonging to the comparator input differential pair were supplied externally to adjust the effective threshold.

\subsection{Gated Oscillator and Delay Block}

With reference to Figure 4, the nominal value of the charging and discharging currents was $2 \mathrm{nA}$ to generate a free-running $1 \mathrm{kHz}$ clock frequency with capacitance $\mathrm{C}_{1}=\mathrm{C}_{2}=\mathrm{C}_{3}=1.1 \mathrm{pF}$. The same values were used in the delay block leading to a $163 \mu$ s delay $\left(\tau_{d}\right)$ for the rising edges of the input data (Din) and $146 \mu \mathrm{s}$ for the falling ones. Since the reset time of the oscillator was $340 \mathrm{~ns}$, the conditions discussed in Section 3 on $\tau_{d}$ were largely satisfied. The start-up time of the oscillator was $\tau_{\text {start-up }}=7 \mu \mathrm{s}$, which implied no preamble was needed for the oscillator to settle. The GO-CDR performances were evaluated by also performing transient noise simulations. The simulated clock rms jitter turned out to be lower than $1 \mu \mathrm{s}$, which was negligible compared with the clock period.

\subsection{Control Logic with Addressing Capabilities}

The CL (Figure 5) was designed and compiled starting from an RTL-HDL behavioral description, targeting a $1.2 \mathrm{~V}$ low-power standard cell library, which yielded a circuit with an 800 equivalent gates complexity. In order to minimize its power consumption, as 
mentioned above, its supply voltage was set to $0.6 \mathrm{~V}$. This required post-layout transistorlevel simulations to verify the correct operation of the circuit. The maximum codeword length and correlator threshold were both set to 16 bits, while the timeout value was set to 63 cycles. This resulted in a 26-bit SIPO register (16 bits for the codeword, 4 bits for the correlator threshold and 6 bits for the timeout value). From the design parameters reported above, it can be concluded that the maximum timeout value limited the maximum packet length to 63 bits. Furthermore, to minimize the preamble time, the CL was designed to detect a start frame delimiter consisting of two consecutive zeros after the first 0-to-1 transition, thus resulting in a 3-bit preamble (100).

\subsection{Bias and Calibration Circuit}

The bias and calibration circuit (Figure 7) was designed to compensate for PVT variations [11]. Assuming temperature variations from $-25^{\circ} \mathrm{C}$ to $+125^{\circ} \mathrm{C}$, and $\pm 12.5 \%$ supply voltage variations, in the worst process corner case, the simulated largest clock frequency error referring to its nominal value $(1 \mathrm{kHz})$ was $15 \%$. The FD and SA Logic were designed and compiled on a $1.2 \mathrm{~V}$ low-power standard cell library. The timeout counter in SA Logic was designed with 8 bits, resulting in 255 clock edges before the raise of the End_Calib signal. The FD and SA Logic yielded a circuit with a 700 equivalent gates complexity.

It was verified through transistor-level simulations that with a $1 \mathrm{kHz}$ Clock_ref, the FD operated correctly for a clock frequency between $700 \mathrm{~Hz}$ and $1450 \mathrm{~Hz}$. Furthermore, it was demonstrated that the timeout value was long enough to enable the FD to detect frequency differences down to $\pm 0.5 \%$. Then, the DCCS was designed using five weighting bits to compensate both clock frequency variations up to $\pm 20 \%$ and calibration loop non-idealities. Simulations demonstrated that the bias and calibration circuit yielded a $\pm 0.5 \%$ GO freerunning frequency accuracy after calibration. This, according to theoretical Equations (1) and (2), resulted in a simulated $N_{m}=100$ bits, which was only affected by the oscillator PVT variations.

During Phase 1, the simulated power consumption of the AFE was $8 \mathrm{nW}$, while that of the baseband logic was $4.8 \mathrm{nW}$, making the total simulated power consumption equal to $12.8 \mathrm{nW}$.

During Phase 2, the average consumption of the baseband logic was $9 \mathrm{nW}$, whereas the AFE still consumed $8 \mathrm{nW}$. Therefore, the total simulated power consumption during Phase 2 was $17 \mathrm{nW}$. Since the operating bitrate was $1 \mathrm{kbps}$, the energy per bit of the proposed system was $17 \mathrm{pJ} /$ bit. The contribution to the overall power consumption of the BC circuit (Figure 7) was not included in this computation because, in the present implementation, Ibias was an external current source. In the final implementation, when Ibias was replaced with the PTAT current generated by the circuit of Figure $3 c$, the simulated additional power consumption of the $\mathrm{BC}$ with a $0.6 \mathrm{~V}$ supply would be $5.48 \mathrm{nW}$, including $0.8 \mathrm{nW}$ consumed by the digital controlled current source.

Figure 8a shows the chip photograph before the application of the protective resin. The AFE occupied $0.2 \mathrm{~mm}^{2}$, whereas the baseband logic area was $0.126 \mathrm{~mm}^{2}$. Most of the overall area was due to passives, in particular the resistors in the $\operatorname{ED}(75 \mathrm{M} \Omega)$ and in the PTAT current generator $\left(13 \mathrm{M} \Omega\right.$ and $113 \mathrm{M} \Omega$ ). An additional area of $0.042 \mathrm{~mm}^{2}$ was due to $\mathrm{BC}$.

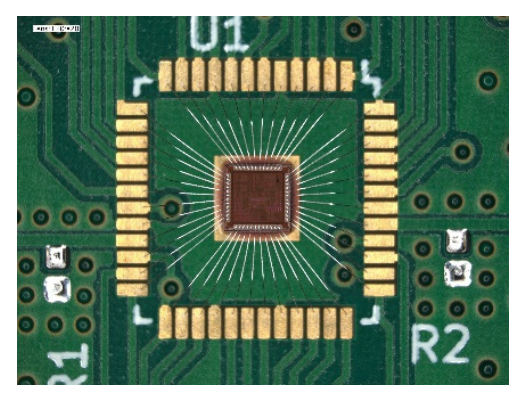

(a)

Figure 8. Cont 


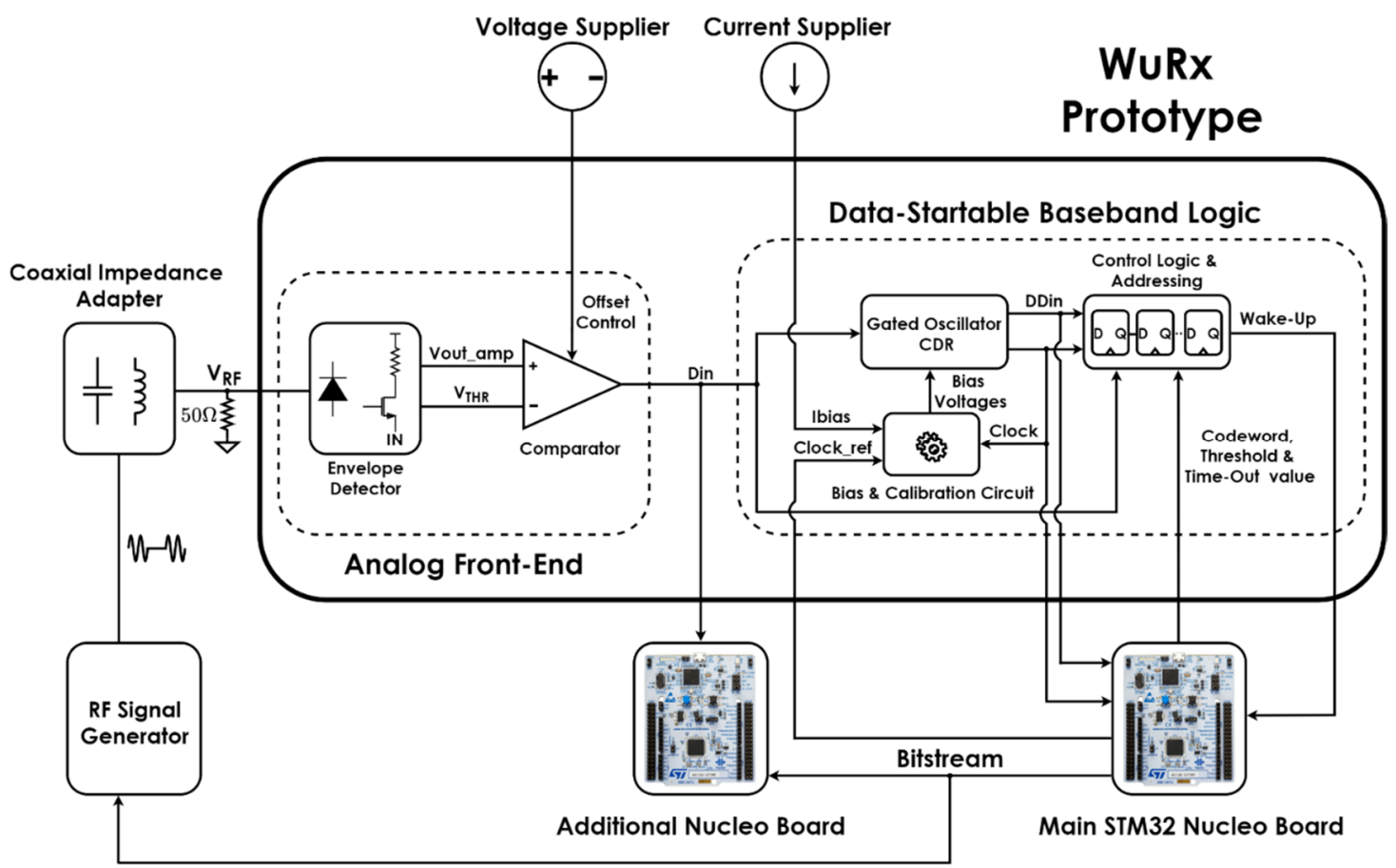

(b)

Figure 8. (a) Chip-on-board photograph. (b) Measurement setup.

\section{Measurement Results}

The fabricated chip was mounted on a board using a chip-on-board wiring technique, as shown in Figure 8a. Figure 8b shows the measurement setup employed for the performance evaluation of the proposed wake-up and data receiver. It included an RF generator for the RF input signal and its OOK modulation. An STM32 Nucleo board (Main Nucleo in Figure $8 b$ ) was used for the generation of the bitstream, programming the SIPO register, processing the output bits generated by the $\mathrm{WuRx}$ and managing the calibration cycle. An additional STM32 Nucleo board, as described below, was used to characterize the impact of the gated-oscillator CDR on the WuRx sensitivity.

The input impedance at the SMA connector was characterized by means of a vector network analyzer (VNA) in the $10 \mathrm{MHz}-1.5 \mathrm{GHz}$ range (see Figure 9). The resonance frequency clearly visible around $1.1 \mathrm{GHz}$ was due to the wire inductance and the input capacitance $(2.95 \mathrm{pF})$, which could be ascribed mainly to the pad, as verified by means of an extracted lumped element equivalent circuit. Indeed, in the present implementation, a standard analog pad was used, which needed to be replaced by a low-capacitance RF pad in the final implementation. Due to these limitations, the present prototype did not address the implementation of the input matching network (IMN). Consequently, all measurements shown hereafter were performed with a $50 \Omega$ resistor soldered parallel to the input of the ED and using a commercial coaxial impedance adapter (see Figure $8 b$ ), thus providing a unity gain IMN. Since the AFE response is independent on the RF carrier frequency, all measurements were performed using the $868 \mathrm{MHz}$ European ISM band carrier frequency.

For the sake of completeness, IMNs for different carrier frequencies were designed using the extracted input impedance lumped element model to estimate the obtainable IMN voltage gain. The simulated IMNs were based on an L-shaped inductor-capacitor (LC) stage using inductances with quality factor $Q=80$ [21]. The simulated IMN gains at $100 \mathrm{MHz}, 433 \mathrm{MHz}$ and $868 \mathrm{MHz}$ were $24.8 \mathrm{~dB}, 17.3 \mathrm{~dB}$ and $8.3 \mathrm{~dB}$, respectively. The simulated IMN gains needed to be added to the measured circuit sensitivity to obtain the projected WuRx total sensitivity. 


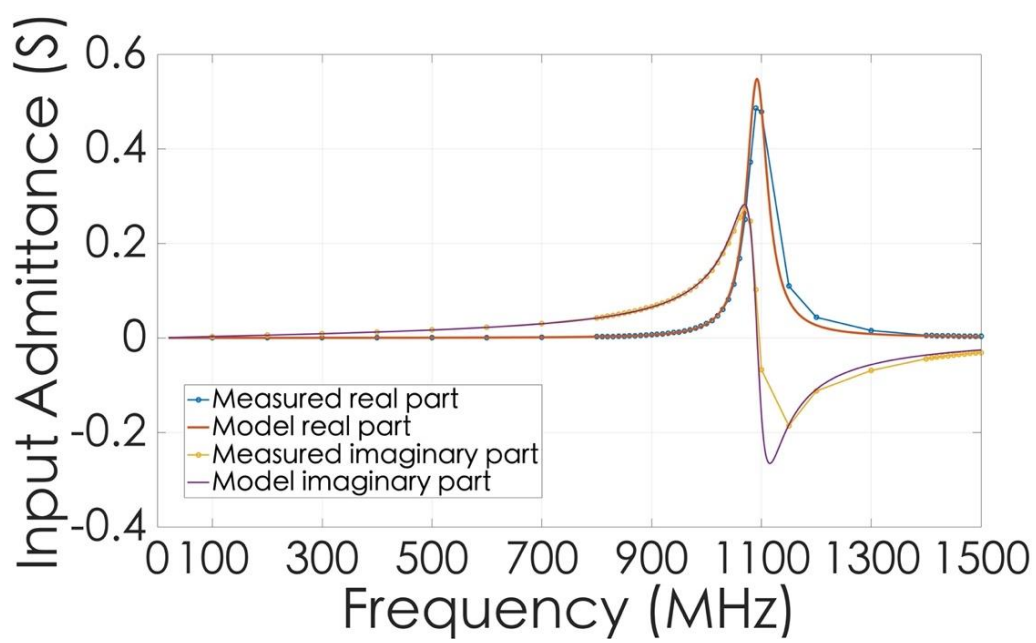

Figure 9. Input admittance vs. frequency. Blue indicates the measured real part of the input admittance, orange is the simulated real part of the input admittance using the extracted model, yellow is the measured imaginary part of the input admittance and violet is the simulated imaginary part of the input admittance using the extracted model.

First, functional tests were performed to verify correct operation. Then, systematic measurements were accomplished to characterize the missed detection rate (MDR) and the false alarm rate (FAR). Finally, the capability of the WuRx to receive long sequences of data was investigated, and the performance of the bias and calibration circuit was analyzed.

The functional tests revealed problems with the data-startable baseband logic, which operated correctly only for a supply voltage ranging from $0.3 \mathrm{~V}$ to $0.5 \mathrm{~V}$ (i.e., lower than the nominal $0.6 \mathrm{~V}$ ). To investigate the precise origin of this unexpected problem, postlayout transistor-level simulations were carried out for different supply voltage values. The simulation results revealed the occurrence of ringing phenomena caused by interline capacitances between the $\mathrm{O} 3$ and Clock signals in Figure 4, which had been underestimated by the extractor. The problem could be suppressed by lowering the supply voltage. Therefore, all measurements shown hereafter have been performed with a $0.4 \mathrm{~V}$ supply for the baseband logic.

Figure 10 shows the sample measured waveforms in response to a packet composed of a 3-bit preamble (100) followed by a 16-bit string matching the stored codeword (1011101101010011). This measurement was performed with a -34 dBm RF input sequence at $1 \mathrm{kbps}$, with a $0.5 \%$ clock frequency error $\left(\left|f_{c k}-f_{b}\right| / f_{b}\right)$ measured after calibration. The curves demonstrate that the ED output was the correct envelope of the modulated RF signal, the generated clock sampled the DDin accurately and the baseband logic correctly generated the wake-up pulse.

MDR measurements were performed to evaluate the sensitivity of the WuRx. The MDR is the ratio between the number of missed wake-ups and the total number of sent packets. To evaluate it, the Nucleo was employed to generate 10,000 equal 19-bit packets (identical to the one reported in Figure 10) separated by $100 \mathrm{~ms}$ from each other and to count the number of wake-up pulses. To investigate the impact of the GO-CDR on the sensitivity of the WuRx, an additional Nucleo (see Figure $8 b$ ), synchronized and running in parallel with the main one, was employed to decode the AFE output (Din, see Figure 1) with an external precisely timed clock and to compare the received stream with the one transmitted by the main Nucleo. The difference between the MDRs computed by the two Nucleo boards was a measure of how far the proposed GO-CDR affected the WuRx sensitivity. The MDR results are reported in Figure 11. The measurements were performed by changing the power of the input RF signal and adjusting the AFE comparator threshold accordingly with a $0.5 \% \mathrm{GO}$ free-running frequency error measured after calibration. Measurements were repeated for correlator thresholds equal to 16/16, 15/16 and 14/16. 


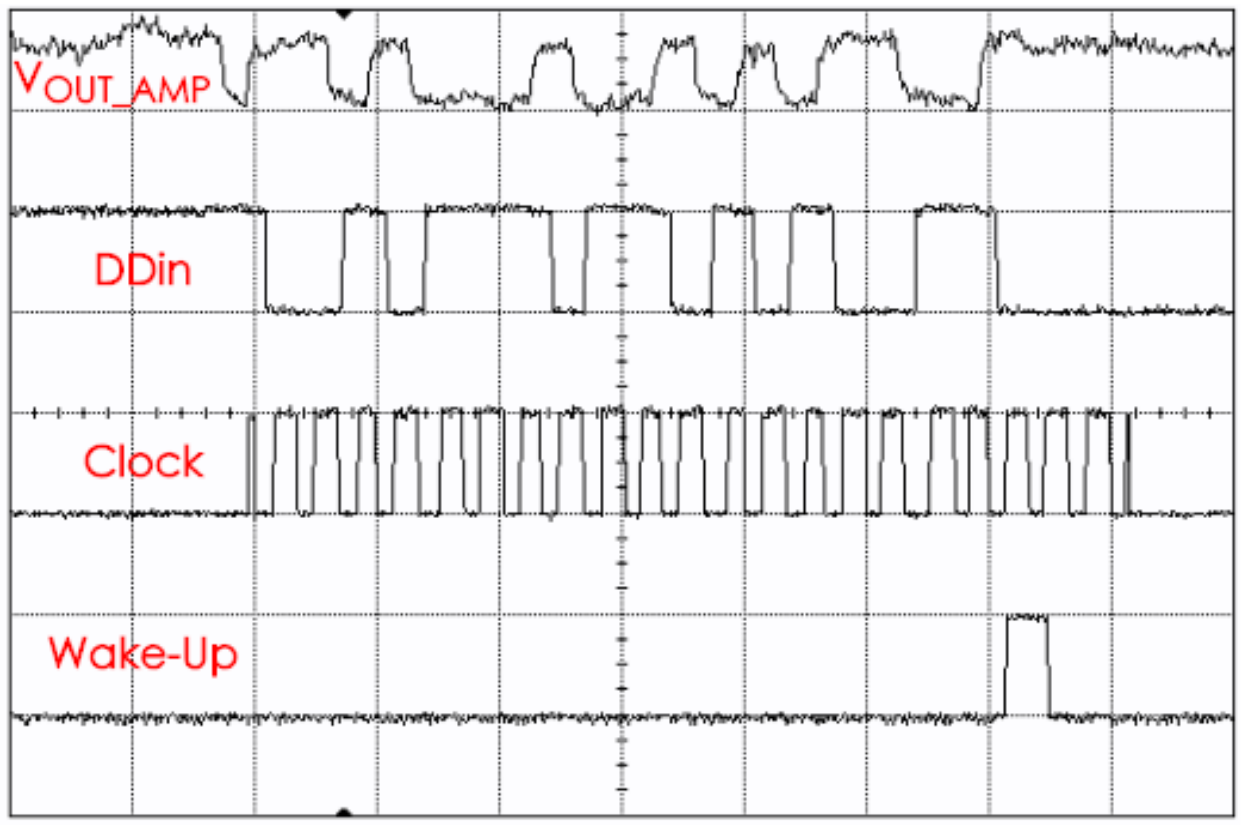

Figure 10. Measured sample waveforms. With reference to Figure 1, from top to bottom: ED output

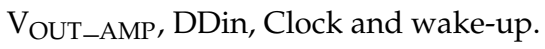

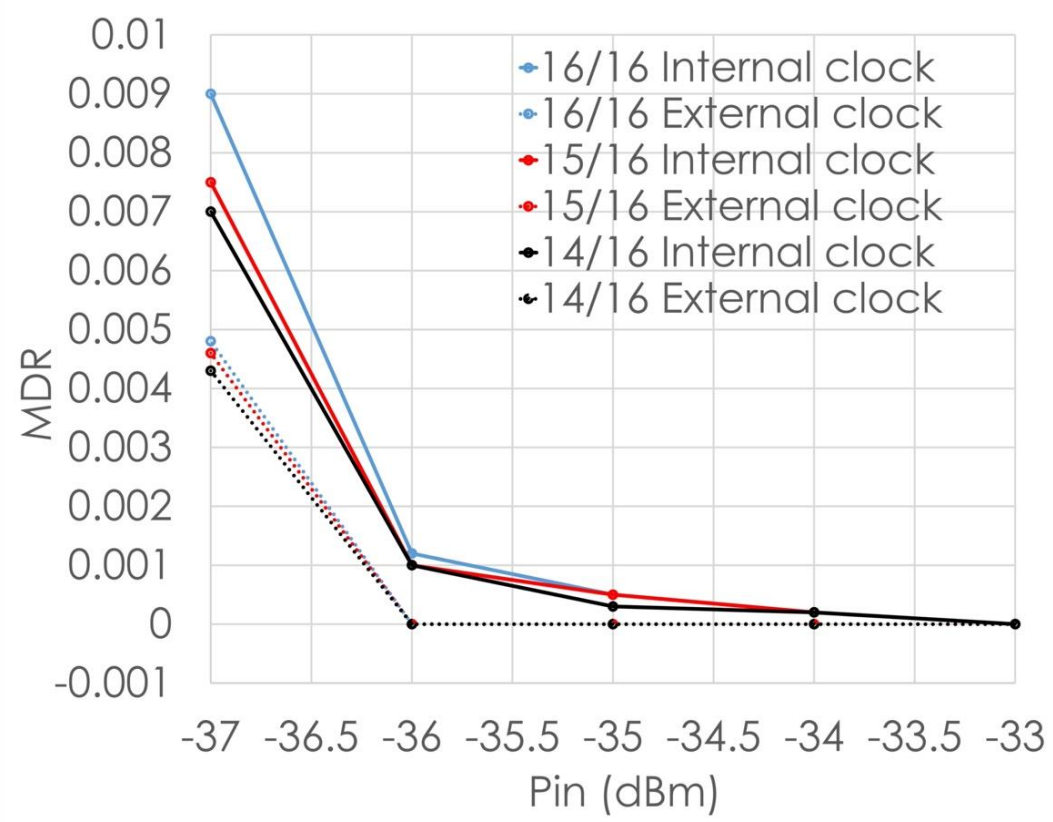

Figure 11. Missed detection rate vs. ED input power. Blue, red and black represent the missed detection rate (MDR) with the correlator threshold set to 16/16, 15/16 and 14/16, respectively. The solid lines are the MDR-measured counts of the wake-up pulses generated by the chip (internal clock). The dotted lines are the MDR-measured decodings of the AFE output stream with an external clock source (external clock).

The Nucleo dedicated to decoding the AFE output was programmed consistently. The input power corresponding to MDR $=10^{-3}$, when the received data was processed by GO-CDR, was $P_{\mathrm{IN}}=-35.75 \mathrm{dBm}$ for the $16 / 16$ case and $\mathrm{P}_{\mathrm{IN}}=-36 \mathrm{dBm}$ for the $14 / 16$ and $15 / 16$ cases. The Nucleo that decoded the Din with an external clock counted an $\mathrm{MDR}=10^{-3}$ for $\mathrm{P}_{\mathrm{IN}}=-36.25 \mathrm{dBm}$ for all correlator thresholds. Therefore, the use of the proposed GO-CDR circuit affected the sensitivity of the WuRx at MDR $=10^{-3}$ for $0.5 \mathrm{dBm}$. 
The same measurement procedure was repeated with different codewords by varying the number of consecutive zeros and ones, the correlator threshold and the codeword length. The measured MDR $=10^{-3}$ was always found for $\mathrm{P}_{\mathrm{IN}}=-35.75 \mathrm{dBm}$ which, as for the aforementioned measurements, was affected by GO-CDR for $0.5 \mathrm{dBm}$.

In the $16 / 16$ case, the total sensitivity at $\mathrm{MDR}=10^{-3}$ referred to the input of the IMN, which included the projected IMN voltage gain, as explained above, of $-60.5 \mathrm{dBm}$, $-53 \mathrm{dBm}$ and $-44 \mathrm{dBm}$ at $100 \mathrm{MHz}, 433 \mathrm{MHz}$ and $868 \mathrm{MHz}$, respectively.

To measure the false alarm rate (FAR), which is defined as the number of false wakeups per hour due to the noise present in the receiver, the input of the coaxial impedance adapter was closed on a $50 \Omega$ resistance. Typically, a FAR $\leq 1 / \mathrm{h}$ is considered acceptable [5]. The Nucleo was used for counting the number of false wake-ups. The correlator was programmed with the 14/16 threshold, the AFE comparator threshold $\mathrm{V}_{\mathrm{THR}}$ was set to the value corresponding to $\mathrm{P}_{\mathrm{IN}}=-35.75 \mathrm{dBm}$, and the clock frequency error measured after calibration was $0.5 \%$. Measurements were performed for $24 \mathrm{~h}$ time windows, resulting in zero overall false wake-ups.

To evaluate the WuRx capability to receive long sequences of data, additional MDR measurements were performed by sending 3174 equal 63-bit packets (for a total of 199,962 transmitted bits) separated by $100 \mathrm{~ms}$ from each other. All the transmitted packets contained a sequence of 20 consecutive ones. As reported in Section 4, the 63-bit packet length was limited in the present prototype by the chosen maximum timeout value of the baseband logic (see Figure 5). To perform these measurements, the output stream of the baseband logic (DDin) was sampled by the main Nucleo, using the clock generated by GO-CDR with a $0.5 \%$ frequency error after calibration (see Figure $8 b$ ). As for the previous MDR measurements on 16-bit codewords, an additional Nucleo was employed to decode the AFE output with an external clock and then to compare the received stream with the one transmitted by the first Nucleo. Measurements were repeated with thresholds on the received bits equal to $63 / 63$ and $58 / 63$. In case the received sequence was processed by GO-CDR, an MDR $=10^{-3}$ was found for $\mathrm{P}_{\mathrm{IN}}=-35 \mathrm{dBm}$ and $\mathrm{P}_{\mathrm{IN}}=-35.5 \mathrm{dBm}$ for the $63 / 63$ and 58/63 cases, respectively. When the received sequence was decoded off-chip by the external MCU clock, an $\mathrm{MDR}=10^{-3}$ was found for $\mathrm{P}_{\mathrm{IN}}=-36.25 \mathrm{dBm}$ in either threshold case. Therefore, the use of the on-chip clock degraded the WuRx sensitivity by $1.25 \mathrm{dBm}$. This measured packet sensitivity differed from the 16-bit code sensitivity in Figure 11 by $0.75 \mathrm{dBm}$, thus demonstrating the GO-CDR capability to also process long data streams. These results lead to the conclusion that the sensitivity is limited by the AFE.

Measurements were repeated in the 63/63 threshold case by varying the number of consecutive zeros and ones from 1 to 63 bits. In any case an MDR $=10^{-3}$ was found with $\mathrm{P}_{\mathrm{IN}}=-35 \mathrm{dBm}$.

Finally, measurements were performed to test the bias and calibration circuit (Figure 7), supplying the $\mathrm{GO}$ with the nominal $\mathrm{V}_{\mathrm{DD}}=0.6 \mathrm{~V}$. The main Nucleo was used to generate the reference clock (Clock_ref) for the frequency detector and manage the control signals (start_calib, end_calib) (see Figure 8b). The current Ibias was set to get an initial frequency error between $-20 \%$ and $+20 \%$ relative to the nominal frequency $(1 \mathrm{kHz})$, and a calibration cycle was performed for each value of Ibias. Figure 12 shows the measured mean frequency error post-calibration, evaluated over 2000 clock periods. The maximum frequency error after calibration was limited to $0.5 \%$, which was consistent with the simulation results. In these conditions, the GO-CDR was tested in terms of the maximum number of equal consecutive bits $\left(N_{m}\right)$. To perform this measurement, 63-bit packets, characterized by a variable number of zeros and ones, were provided to GO-CDR (i.e., excluding the AFE) by the Nucleo. The same Nucleo was used to sample the DDin using the clock generated by GO-CDR. The measurements revealed $N_{m}=63$ bits, thus demonstrating that GO-CDR was able to process packets even in case where they were made of all zeros or ones. With a $1 \%$ clock frequency error, $N_{m}$ decreased to 50 bits. These results were consistent with both Equations (1) and (2) and the simulation results, which projected $N_{m} \sim 100$ bits and 50 bits with $\alpha=0.005$ and 0.01 , respectively (i.e., far above the maximum packet length 
of the WuRx). Furthermore, $N_{m}$ was not affected by the noise in the GO. The clock rms jitter was found to be $3 \mu \mathrm{s}$, thus revealing that $N_{m}$ was only affected by the free-running GO-CDR frequency error.

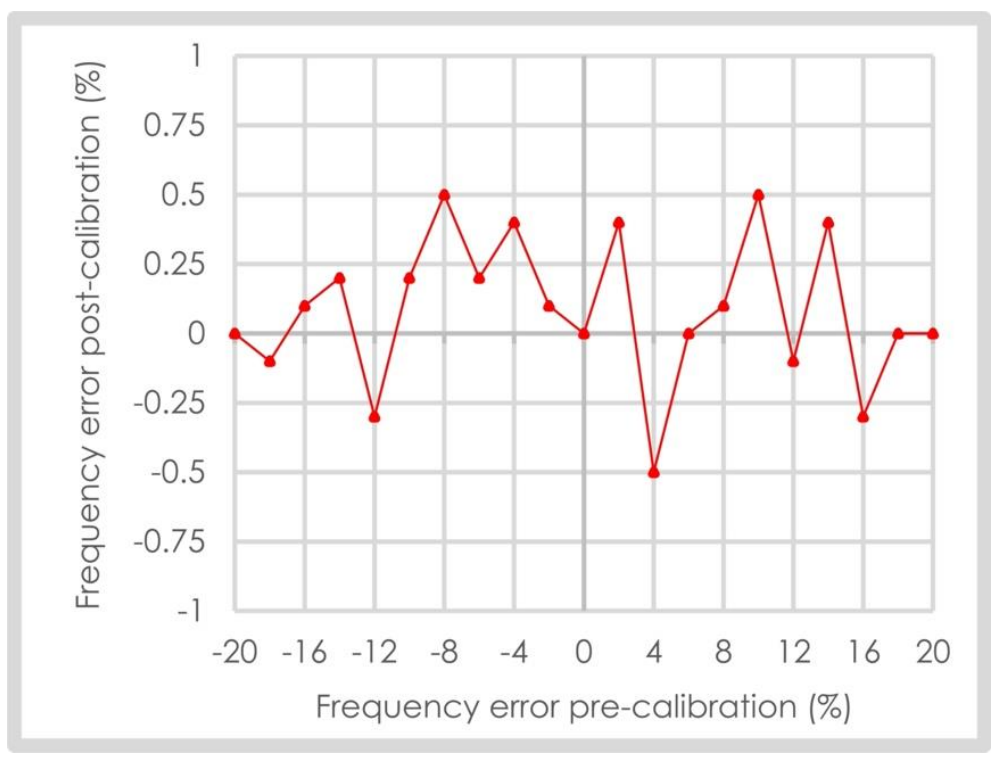

Figure 12. Post-calibration vs. pre-calibration GO frequency error.

\section{Discussion and Conclusions}

This paper presented a nanowatt WuRx which enabled nodes to receive long data streams in addition to a wake-up codeword. It included an always-on clockless AFE and a data-startable baseband logic based on a gated oscillator clock and data recovery (GO-CDR) circuit. GO-CDR ensured phase alignment between the received data and clock with nanowatt power consumption, thus avoiding the use of power-hungry PLLs or crystal oscillators. Any free-running frequency mismatch between the GO and bitrate did not limit the number of receivable bits, but rather only the maximum number of receivable equal consecutive bits $\left(N_{m}\right)$. To overcome this limitation, the proposed system included a frequency calibration circuit.

The proposed architecture was fabricated in STMicroelectronics $90 \mathrm{~nm} \mathrm{BCD}$ technology. The circuit was supplied with $0.6 \mathrm{~V}$, and the overall power consumption, excluding the calibration circuit, was $12.8 \mathrm{nW}$ during the rest state and $17 \mathrm{nW}$ at a $1 \mathrm{kbps}$ data rate. Measurements on the GO-CDR calibration circuit revealed that, starting from a $\pm 20 \%$ initial error, the maximum free-running frequency error after calibration was $\pm 0.5 \%$. In these conditions, the GO-CDR correctly sampled packets even if they were made of all zeros or ones. In the same conditions, with a $100 \mathrm{MHz}$ RF carrier $1 \mathrm{kbps}$ OOK modulated input, a $10^{-3}$ missed detection rate (MDR) with a $-60.5 \mathrm{dBm}$ sensitivity (including the projected input matching network gain) was measured, transmitting 16-bit codewords and tolerating 0 errors. The WuRx sensitivity was mainly limited by the AFE. A comparison with an experimental setup where sampling and correlation were performed by an external MCU with precise clock showed that the GO-CDR reduced the WuRx sensitivity by $0.5 \mathrm{dBm}$. Furthermore, it has been verified through measurements that WuRx received, with MDR $=10^{-3}, 63$-bit packets, even if they were made of all zeros or ones, with a 0-bit error tolerance and a $-59.8 \mathrm{dBm}$ sensitivity (including the projected input matching network gain). In this case, the GO-CDR affected the sensitivity for $1.25 \mathrm{dBm}$. Finally, the WuRx false alarm rate (FAR) was measured for $24 \mathrm{~h}$ time windows, resulting in zero overall false wake-ups.

Table 1 summarizes the system performance and compares it with other state-of-theart WuRxs reported in the literature. When we compare the Figure-of-Merit (FoM), which is conventionally defined to take into account the sensitivity normalized to the bitrate 
and the power consumption, it can be observed that our implementation provided similar performance compared to other state-of-the-art WuRxs. However, it must be remarked that the sensitivity is determined essentially by the AFE, which is not the main focus of this paper. Therefore, we do not comment further on this point.

Table 1. Comparison with state-of-the-art wake-up receivers.

\begin{tabular}{|c|c|c|c|c|c|c|}
\hline & \multicolumn{2}{|c|}{ This Work } & $\begin{array}{c}\text { [17] } \\
\text { ESSCIRC'19 }\end{array}$ & $\begin{array}{c}{[22]} \\
\text { JSSC'19 }\end{array}$ & $\begin{array}{c}{[5]} \\
\text { ISSCC'19 }\end{array}$ & $\begin{array}{c}\text { [9] } \\
\text { TMTT'20 }^{\prime}\end{array}$ \\
\hline Wake-up and/or data & \multicolumn{2}{|c|}{ Wake-up + data } & Wake-up + data & Wake-up & Wake-up & Wake-Up \\
\hline RF frequency (MHz) & 100 & 433 & 750 & $151.8^{1}$ & $434.4^{1}$ & 2200 \\
\hline Bitrate (kbps) & \multicolumn{2}{|c|}{1} & 200 & 0.2 & 0.1 & 0.250 \\
\hline Technology (nm) & \multicolumn{2}{|c|}{90} & 65 & 130 & 65 & 65 \\
\hline Voltage supply (V) & \multicolumn{2}{|c|}{0.6} & 0.4 & $1-0.6$ & 0.4 & $1-0.5$ \\
\hline Power in listening (nW) & \multicolumn{2}{|c|}{12.8} & 1484 & 7.4 & 0.42 & 28.2 \\
\hline $\begin{array}{l}\text { Power in listening }+1 \% \\
\text { reception }{ }^{(1)}(\mathrm{nW})\end{array}$ & \multicolumn{2}{|c|}{12.9} & 1486 & 7.4 & 0.42 & 28.2 \\
\hline IMN gain $(\mathrm{dB})$ & $24.8^{(2)}$ & $17.3^{(2)}$ & 13 & 27 & 23 & 12.6 \\
\hline Sensitivity (dBm) & $-59.8^{(3)(4)}$ & $-52.3^{(3)(4)}$ & $-50^{(3)}$ & $-76^{(3)}$ & $-79.1^{(3)}$ & $-68^{(5)}$ \\
\hline Normalized sensitivity ${ }^{(6)}(\mathrm{dB})$ & $-74.8^{(4)}$ & $-67.3^{(4)}$ & -76.5 & -87.5 & -89.1 & -80 \\
\hline $\operatorname{FoM}^{(7)}(\mathrm{dB})$ & $-123.7^{(4)}$ & $-116.2^{(4)}$ & -104.2 & -138.8 & -152.9 & -125.5 \\
\hline Synchronization technique & \multicolumn{2}{|c|}{ GO-CDR } & Data-locked Osc. & $\begin{array}{l}\text { Phase-shifted RF } \\
\text { transmission }(8)\end{array}$ & Oversampling & Oversampling \\
\hline Maximum packet length & \multicolumn{2}{|c|}{$63^{(9)}$} & 40 & 8 & 11 & 63 \\
\hline Error tolerance (bit) & \multicolumn{2}{|c|}{0} & 0 & 3 & 1 & 13 \\
\hline $\begin{array}{l}\text { Maximum number of equal } \\
\text { consecutive bits }\end{array}$ & \multicolumn{2}{|c|}{63} & $\mathrm{~N} / \mathrm{A}$ & 8 & 11 & 63 \\
\hline
\end{tabular}

(1) Computed assuming 1\% activity of reception [15]. (2) Input matching network (IMN) gains of this paper are estimated through simulations. (3) Sensitivity defined through a $10^{-3}$ missed detection rate (MDR). In this paper it was evaluated using 63-bit packets. ${ }^{(4)}$ Includes the IMN gains estimated through simulations. ${ }^{(5)}$ Sensitivity defined through a 0.02 missed detection rate (MDR). ${ }^{\left({ }^{6}\right)}$ Normalized sensitivity $=$ Sensitivity $-5 \log B W_{\mathrm{BB}}$, where $\mathrm{BW}_{\mathrm{BB}}=$ bitrate (derived from [8]). ${ }^{(7)}$ FoM $=$ Normalized sensitivity $+10 \log ($ Power $/ 1 \mathrm{~mW})$. (8) A half clock cycle phase-shifted RF transmission is sent after the initial transmission to protect against TX/WuRx asynchronization [20].

(9) Maximum packet length is only limited by the maximum timeout value.

Table 1 shows that the proposed WuRx provides state-of-the-art performance in terms of the maximum packet length, error tolerance and maximum number of equal consecutive bits. Oversampling techniques, such as those in [5] and [9], exhibit limitations on the maximum packet length (11 bits and 63 bits, respectively) but do not set a constraint on $N_{m}$. It must be noticed that [9] showed the only WuRx which achieved the same packet length as the wake-up and data receiver we propose (i.e., 63 bits). In Reference [9], a 13-bit error tolerance was accepted, while in our implementation, the same packet length was achieved with 0 errors and was only limited by the timeout register size. Furthermore, in [9], the sensitivity was evaluated with $\mathrm{MDR}=20 \times 10^{-3}$ and $\mathrm{FAR}<1 / \mathrm{h}$, while as reported above, we characterized the performance of the proposed $\mathrm{WuRx}$ with more stringent constraints (i.e., $\mathrm{MDR}=10^{-3}$ and FAR $=0$ ).

In conclusion, we believe that the proposed scheme is well suited for ultra-low-power WuRxs with the capability to receive long streams.

Author Contributions: Conceptualization, M.D., A.M.E., L.P., E.F.S., A.G., R.C. and G.R.; Investigation, M.D., A.M.E., L.P., E.F.S., A.G., R.C. and G.R.; Methodology, M.D., A.M.E., L.P., E.F.S., A.G., R.C. and G.R.; Supervision, E.F.S., A.G., R.C. and G.R.; Validation, M.D., A.M.E., L.P., E.F.S., A.G., R.C. and G.R.; Writing-original draft, M.D., A.M.E., L.P., E.F.S., A.G., R.C. and G.R.; Writing-review \& editing, M.D., A.M.E., L.P., E.F.S., A.G., R.C. and G.R. All authors have read and agreed to the published version of the manuscript.

Funding: This research received no external funding.

Conflicts of Interest: The authors declare no conflict of interest. 


\section{References}

1. Perilli, L.; Scarselli, E.F.; la Rosa, R.; Canegallo, R. Wake-Up Radio Impact in Self-Sustainability of Sensor and Actuator Wireless Nodes in Smart Home Applications. In Proceedings of the 2018 Ninth International Green and Sustainable Computing Conference (IGSC), Pittsburgh, PA, USA, 22-24 October 2018; pp. 1-7.

2. Magno, M.; Jelicic, V.; Srbinovski, B.; Bilas, V.; Popovici, E.; Benini, L. Design, Implementation, and Performance Evaluation of a Flexible Low-Latency Nanowatt Wake-Up Radio Receiver. IEEE Trans. Ind. Inform. 2016, 12, 633-644. [CrossRef]

3. Oh, S.; Roberts, N.E.; Wentzloff, D.D. A $116 \mathrm{nW}$ multi-band wake-up receiver with 31-bit correlator and interference rejection. In Proceedings of the IEEE 2013 Custom Integrated Circuits Conference, San Jose, CA, USA, 23-25 September 2013; pp. 1-4.

4. $\quad$ Roberts, N.E.; Craig, K.; Shrivastava, A.; Wooters, S.N.; Shakhsheer, Y.; Calhoun, B.H.; Wentzloff, D.D. 26.8 A 236 nW-56.5 $\mathrm{dBm}$-sensitivity bluetooth low-energy wakeup receiver with energy harvesting in $65 \mathrm{~nm}$ CMOS. In Proceedings of the 2016 IEEE International Solid-State Circuits Conference (ISSCC), San Francisco, CA, USA, 5-9 February 2016; pp. 450-451.

5. Mangal, V.; Kinget, P.R. 28.1 A $0.42 \mathrm{nW} 434 \mathrm{MHz}-79.1 \mathrm{dBm}$ Wake-Up Receiver with a Time-Domain Integrator. In Proceedings of the 2019 IEEE International Solid-State Circuits Conference (ISSCC), San Francisco, CA, USA, 17-21 February 2019; pp. 438-440.

6. Mangal, V.; Kinget, P.R. A Wake-Up Receiver with a Multi-Stage Self-Mixer and with Enhanced Sensitivity When Using an Interferer as Local Oscillator. IEEE J. Solid State Circuits 2019, 54, 808-820. [CrossRef]

7. Moody, J.; Bassirian, P.; Roy, A.; Liu, N.; Pancrazio, S.; Barker, N.S.; Calhoun, B.H.; Bowers, S.M. A -76 dBm 7.4 nW wakeup radio with automatic offset compensation. In Proceedings of the 2018 IEEE International Solid-State Circuits Conference (ISSCC), San Francisco, CA, USA, 11-15 February 2018; pp. 452-454.

8. Wang, P.P.; Jiang, H.; Gao, L.; Sen, P.; Kim, Y.; Rebeiz, G.M.; Mercier, P.P.; Hall, D.A. A Near-Zero-Power Wake-Up Receiver Achieving -69-dBm Sensitivity. IEEE J. Solid State Circuits 2018, 53, 1640-1652. [CrossRef]

9. Bassirian, P.; Duvvuri, D.; Liu, N.; Tsao, D.T.H.; Barker, N.S.; Calhoun, B.H.; Bowers, S.M. Design of an S-Band Nanowatt-Level Wakeup Receiver with Envelope Detector-First Architecture. IEEE Trans. Microw. Theory Tech. 2020, 68, 3920-3929. [CrossRef]

10. Elhoseny, M.; Hassanien, A.E. Secure Data Transmission in WSN: An overview. In Dynamic Wireless Sensor Networks; Springer: Berlin/Heidelberg, Germany, 2019; pp. 115-143.

11. D'Addato, M.; Antolini, A.; Renzini, F.; Elgani, A.M.; Perilli, L.; Scarselli, E.F.; Gnudi, A.; Magno, M.; Canegallo, R. Nanowatt Clock and Data Recovery for Ultra-Low Power Wake-Up Receivers. In Proceedings of the 2020 International Conference on Embedded Wireless Systems and Networks (EWSN 2020), Lyon, France, 17-19 February 2020; pp. 224-229.

12. Huang, K.-K.; Brown, J.K.; Collins, N.; Sawyer, R.K.; Yahya, F.B.; Wang, A.; Roberts, N.E.; Calhoun, B.H.; Wentzloff, D.D. A Fully Integrated $2.7 \mu \mathrm{W}-70.2 \mathrm{dBm}$-Sensitivity Wake-Up Receiver with Charge-Domain Analog Front-End. 16.5 dB-SIR, FEC and Cryptographic Checksum. In Proceedings of the 2021 IEEE International Solid-State Circuits Conference (ISSCC), San Francisco, CA, USA, 13-22 February 2021; pp. 306-308.

13. Bishop, H.L.; Dissanayake, A.; Bowers, S.M.; Calhoun, B.H. 21.5 An Integrated 2.4 GHz-91.5 dBm-Sensitivity Within-Packet Duty-Cycled Wake-Up Receiver Achieving $2 \mu \mathrm{W}$ at $100 \mathrm{~ms}$ Latency. In Proceedings of the 2021 IEEE International Solid-State Circuits Conference (ISSCC), San Francisco, CA, USA, 13-22 February 2021; pp. 310-312.

14. D'Addato, M. Progetto di un PLL a Bassissimo Consumo per Sistemi Wake-Up Radio. Master's Thesis, Department Electronic Engineering Bologna University, Bologna, Italy, 2019.

15. Chen, S.; Lin, J.; Li, H.; Cheng, K. Reference-less wake-up receiver with noise suppression and injection-locked clock recovery. IET Circuits Devices Syst. 2020, 14, 168-175. [CrossRef]

16. Dissanayake, A.; Moody, J.; Bishop, H.L.; Truesdell, D.; Muhlbauer, H.; Lu, R.; Gao, A.; Gong, S.; Calhoun, B.H.; Bowers, M.S. A $-108 \mathrm{dBm}$ Sensitivity, $-28 \mathrm{~dB}$ SIR, $130 \mathrm{nW}$ to $41 \mu \mathrm{W}$, Digitally Reconfigurable Bit-Level Duty-Cycled Wakeup and Data Receiver. In Proceedings of the 2020 IEEE Custom Integrated Circuits Conference (CICC), Boston, MA, USA, 25-30 April 2020; pp. 1-4.

17. Elhebeary, M.; Chen, L.; Pamarti, S.; KenYang, C. An 8.5 pJ/bit Ultra-Low Power Wake-Up Receiver Using Schottky Diodes for IoT Applications. In Proceedings of the ESSCIRC 2019-IEEE 45th European Solid-State Circuits Conference (ESSCIRC), Cracow, PL, USA, 23-26 September 2019; pp. 205-208.

18. Elgani, A.M.; Renzini, F.; Perilli, L.; Scarselli, E.F.; Gnudi, A.; Canegallo, R.; Ricotti, G. A Clockless Temperature-Compensated Nanowatt Analog Front-End for Wake-Up Radios Based on a Band-Pass Envelope Detector. IEEE Trans. Circuits Syst. I Regul. Pap. 2020, 67, 2612-2624. [CrossRef]

19. Brokaw, A. A simple three-terminal IC bandgap reference. In Proceedings of the 1974 IEEE International Solid-State Circuits Conference. Digest of Technical Papers, Philadelphia, PA, USA; 1974; pp. 188-189. [CrossRef]

20. Assaad, M.; Alser, M.H. Design of an all-digital synchronized frequency multiplier based on a dual-loop (D/FLL) architecture. In Proceedings of the 2012 IEEE Symposia on VLSI Technology and Circuits (VLSI), Honolulu, HI, USA, 13-19 June 2012; pp. 1-7.

21. Mangal, V.; Kinget, P.R. Sub-nW Wake-Up Receivers with Gate-Biased Self-Mixers and Time-Encoded Signal Processing. IEEE J. Solid State Circuits 2019, 54, 3513-3524. [CrossRef]

22. Moody, J.; Bassirian, P.; Roy, A.; Liu, N.; Barker, N.S.; Cahloun, B.H.; Bowers, S.M. Interference Robust Detector-First Near-Zero Power Wake-Up Receiver. IEEE J. Solid State Circuits 2019, 54, 2149-2162. [CrossRef] 